\title{
Prevalence, Clinical Characteristics, and Outcomes Related to Ventilator-Associated Events in Neurocritically III Patients
}

\author{
Venus Kit Sze Wu' ${ }^{1}$, Christine Fong ${ }^{2}$, Andrew M. Walters ${ }^{2}$ and Abhijit V. Lele $2,3,4,5^{*}$ \\ C 2020 Springer Science+Business Media, LLC, part of Springer Nature and Neurocritical Care Society
}

\begin{abstract}
Background: The prevalence, characteristics, and outcomes related to the ventilator-associated event(s) (VAE) in neurocritically ill patients are unknown and examined in this study.

Methods: A retrospective study was performed on neurocritically ill patients at a 413-bed level 1 trauma and stroke center who received three or more days of mechanical ventilation to describe rates of $V A E$, describe characteristics of patients with VAE, and examine the association of VAE on ventilator days, mortality, length of stay, and discharge to home.

Results: Over a 5-year period from 2014 through 2018, 855 neurocritically ill patients requiring mechanical ventilation were identified. A total of 147 VAEs occurred in 130 (15.2\%) patients with an overall VAE rate of 13 per 1000 ventilator days and occurred across age, sex, BMI, and admission Glasgow Coma Scores. The average time from the start of ventilation to a VAE was 5 (range 3-48) days after initiation of mechanical ventilation. Using Centers for Disease Control and Prevention definitions, VAEs met criteria for a ventilator-associated condition in $58 \%$ of events $(n=85)$, infection-related VAE in $22 \%$ of events $(n=33)$, and possible ventilator-associated pneumonia in $20 \%$ of events $(n=29)$. A most common trigger for VAE was an increase in positive end-expiratory pressure (84\%). Presence of a VAE was associated with an increase in duration of mechanical ventilation (17.4[IQR 20.5] vs. 7.9[8.9] days, $p<0.001$, 95\% Cl 7.86-13.92), intensive care unit (ICU) length of stay (20.2[1.1] vs. 12.5[0.4] days, $p<0.00195 \%$ Cl 5.3-10.02), but not associated with in-patient mortality (34.1 vs. $31.3 \%$. 95\% Cl 0.76-1.69) or discharge to home (12.7\% vs. 16.3\%, 95\% 0.47-1.29).

Conclusions: VAE are prevalent in the neurocritically ill. They result in an increased duration of mechanical ventilation and ICU length of stay, but may not be associated with in-hospital mortality or discharge to home.
\end{abstract}

Keywords: Ventilator-associated event, VAE, Neurocritical care, Outcomes, Mechanical ventilation

\section{Introduction}

The National Healthcare Safety Network implemented ventilator-associated event (VAE) surveillance in multiple US hospitals starting in January 2003 [1]. Recent

\footnotetext{
*Correspondence: abhijit2@uw.edu

${ }^{2}$ Department of Anesthesiology and Pain Medicine, University of Washington, Seattle, WA, USA

Full list of author information is available at the end of the article
}

studies have suggested that VAE, as a broader marker for inflammation, may be the better outcomes metric than ventilator-associated pneumonia (VAP) in critically ill trauma patients [2]; however, this has not been validated nor tested in the neurocritically ill patients.

The incidence of mechanical ventilation in patients admitted to neurological and neurosurgical intensive care units is estimated to be $30-35 \%$ and may be higher than patients in other critical care units [3, 4]. After a period of stability, change in positive end-expiratory

The study was conducted at Harborview Medical Center. 
pressure (PEEP), or fraction of delivered oxygen (FiO2) constitutes a VAE. Under the VAE surveillance tool are three nested tiers. A ventilator-associated condition (VAC) comprises the first tier and includes all patients with clinically significant pulmonary complications, irrespective of whether inflammation or infection may be the contributing factors. The second tier, infection-related ventilated-associated complications (IVAC), is reached when VAC criteria are met in addition to signs of leukocytosis, abnormal temperature, and commencement of antimicrobials. Progression to the third tier occurs when evidence exists of a pulmonary source of infection and is called probable or possible ventilator-associated pneumonia (PVAP) (Supplementary Figure 1) [1].

The rates of VAE among subgroups of patients such as isolated traumatic brain or spinal cord injury, non-traumatic subarachnoid or intraparenchymal hemorrhage, and acute ischemic stroke are unknown. Additionally, characteristics of patients, types, and triggers of VAE, and the association between VAE and outcomes such as neurocritical care unit and hospital length of stay, inhospital mortality, and discharge to home have not previously been described.

Thus, in this study of neurocritically ill patients, we aimed to (1) describe the prevalence, types, and triggers of VAE, (2) describe characteristics of patients with and without VAE, and (3) examine clinical outcomes (intensive care unit and hospital length of stay, mortality, and discharge to home) in patients with VAE.

\section{Methods}

\section{Study Design and Study Population}

This retrospective cohort study was conducted at Harborview Medical Center, a 413-bed level 1 trauma, and a comprehensive stroke center. Patients with various neurological emergencies such as non-traumatic subarachnoid hemorrhage, intracerebral hemorrhage, isolated traumatic brain, isolated spinal cord injury, acute ischemic stroke, neuromuscular disorders, and those requiring intensive care monitoring after surgical and interventional procedures are admitted to a dedicated 30-bed neurocritical care unit which is staffed by board-certified intensivists, resident and fellow trainees, advanced practice providers and nocturnal intensivists.

Patients who were admitted to the neurocritical care service between January 1, 2014, and December 31, 2018, and who were mechanically ventilated for three or more days [2] were included in this study.

\section{Data Sources}

Patient data for this study were extracted from the enterprise clinical data warehouse (EDW) at the University of Washington. We identified neurocritically ill patients admitted to the Neurocritical Care Service at Harborview Medical Center by querying admit and transfer orders. We then extracted hospitalization and outcome data for these patients from the EDW using various data sources, including hospital registration, nursing charts, clinical notes, orders, and facility billing data. We want to acknowledge the Center for Perioperative and Pain Initiatives in Quality Safety Outcome at the University of Washington for providing data for this study.

\section{Exposures and Outcomes}

The exposures of interest were patient characteristics, including age, sex, body mass index, diagnosis, Glasgow Coma Score (GCS), and intracranial pressure, fluid balance during the time spent on mechanical ventilation.

The primary outcomes were the prevalence of VAE and type and triggers for VAE by subgroups of patients with isolated traumatic and spinal cord injury, nontraumatic subarachnoid hemorrhage, and intraparenchymal hemorrhage, acute ischemic stroke. We defined VAE and its subgroups, VAC, IVAC, and PVAP, in accordance with the Centers for Disease Control and Prevention's (CDC-NHSN) guidelines (Supplementary Figure 1) [5, 6]. A VAE was diagnosed as having at least 2 days of mechanical ventilation with a stable or decreasing FIO2 or PEEP, followed by an increase in PEEP $(>3 \mathrm{cmH} 2 \mathrm{O})$ or FIO2 $(\geq 0.20)$ for at least two subsequent days. As such, we only included patients mechanically ventilated for at least 3 days $[2,6]$.

The secondary outcomes were total duration on mechanical ventilation, intensive care unit, and hospital length of stay, in-hospital mortality, and discharge to home.

Our neurocritical care unit standards include initiation of enteral nutrition to all patients within 24-48 h of admission, ventilator weaning strategies including daily spontaneous breathing trials and aggressive titration of FiO2 and PEEP to as low levels as tolerable to maintain $\mathrm{SpO} 2$ greater or equal to $92 \%$, and interruption in sedation for neurological wake-up tests for serial "q1 hour" neurological examinations. Our institutional infection control program performs electronic screening of the medical record for patients with VAE as described above.

\section{Statistical Analysis \\ Rate of VAE}

Overall and condition-specific VAE rates were calculated as events per 1000 ventilator days. Also, rates of VAC, IVAC, and PVAP were calculated as events per 1000 ventilator days. 


\section{Description of Characteristics of Patients with and without VAE}

The study sample was described using mean (standard deviation) or median (interquartile range, IQR), whenever appropriate. We describe (as count and percentage) the characteristics of patients such as age, sex, body mass index, diagnosis, admission GCS, intracranial pressure, fluid balance during the time spent on mechanical ventilation, sedative (propofol, dexmedetomidine, midazolam, pentobarbital), and any neuromuscular blocker infusions in the VAE and non-VAE groups. For age, body mass index (BMI), intracranial pressures, admission GCS, partial pressure of oxygen (PO2), partial pressure of carbon dioxide (PCO2), $\mathrm{FiO} 2$ and Partial pressure of oxygen/Fraction of inspired oxygen $(\mathrm{PaO} 2 / \mathrm{FiO} 2)$ ratio, intensive unit length of stay, hospital length of stay, duration of mechanical ventilation, we conducted a 2-tailed $t$ test to calculate the difference between the mean and calculated $p$ value and $95 \%$ confidence intervals. For the categorical variables (male sex, positive fluid balance while on mechanical ventilation, sedative and neuromuscular blockade, mortality, the transition to comfort measures, and discharge to home) we conducted Fisher's exact test and calculated relative risk (95\% confidence interval) for development of VAE. Stata 15.1 [7] was used for statistical analysis.

The study (Study Number 00006414) was approved by the Institutional Review Board of the University of Washington.

\section{Results}

\section{Sample Characteristics}

Out of 7055 neurocritically ill patients, 2827 (40.1\%) patients were mechanically ventilated between January 1, 2014, and December 31, 2018 (Fig. 1). Of these, 855 $(30.2 \%)$ received mechanical ventilation for three or more days and comprised the final study sample (Table 1).

\section{Prevalence, Types, and Triggers for VAEs Prevalence of VAES}

A total of 147 VAEs occurred in 130 (15.2\%) patients (Table 2). The overall rate for the development of VAE was 13 per 1000 ventilator days. The lowest VAE rate was in patients admitted to the intensive care unit after elective neurosurgical procedures (5.58 per 1000 ventilator days). The highest rate of VAE was among patients with status epilepticus (26.98 per 1000 ventilator days).

\section{Types and Triggers for VAE}

VAEs were classified as a VAC in $58 \%$ of events $(n=85)$. They were further classified as an IVAC in $22 \%$ of events $(n=33)$. Finally, $20 \%(n=29)$ of VAEs met criteria for PVAP (Table 3). Change in PEEP was the most common

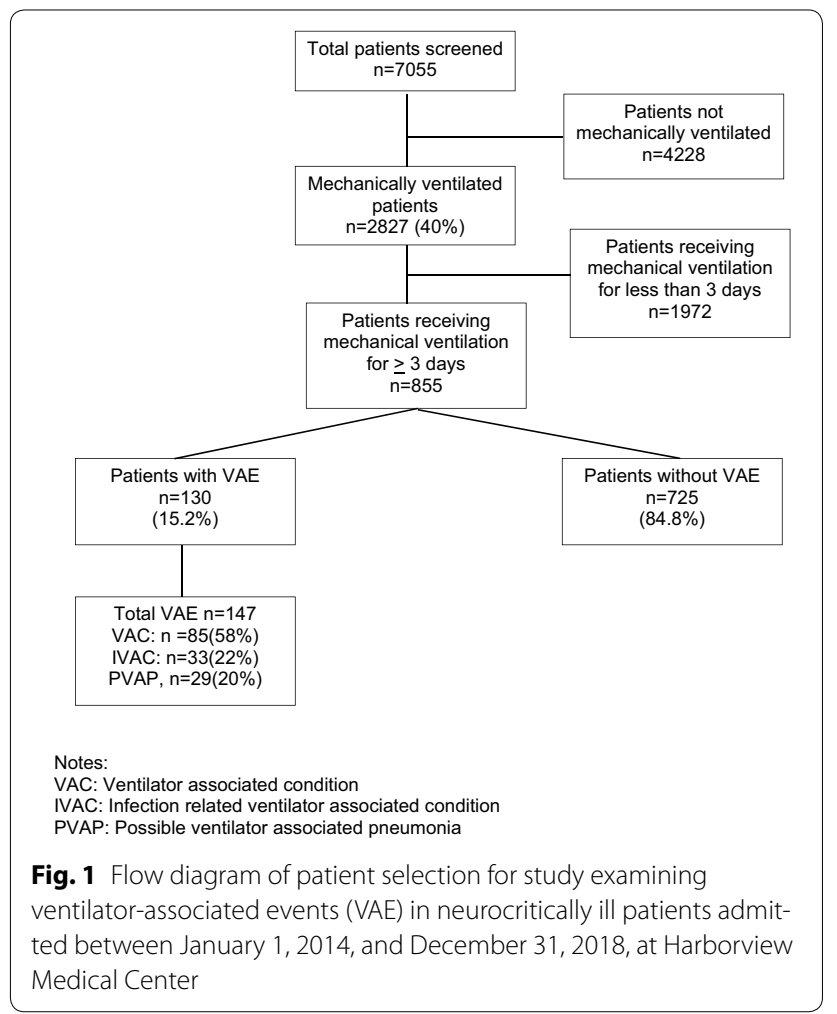

trigger for VAE occurring in 123/147 (83.7\%), with the remaining $24 / 147(16.3 \%)$ being triggered by a change in $\mathrm{FiO} 2$.

\section{Characteristics of Patients with and without VAE}

Demographics Age (52[SD 18.4] vs. 56.6[SD 17.03] years, 95\% CI 0.51-7.35, male sex (70\% vs. 61\%, 95\% CI 1.011.31), BMI (29.8[SD 8.6] vs. 27.9[SD 7], 95\% 0.49-3.29), and admission GCS (9 [range 3-15] vs. 7 [range 3-15], 95\% CI 0.4-1.36) were comparable between the VAE and non-VAE groups (Table 1).

Overall, $31 \%$ of patients with VAE and $40.2 \%$ of patients without VAE received an intracranial pressure monitor. The maximum intracranial pressure was $10.6 \mathrm{mmHg}$ higher in patients with VAE (39.6[SD 23.7] $\mathrm{mmHg}$ vs. 29[SD 18.4] mmHg, $p<0.001,95 \%$ CI 5.3-15.8).

Analgosedation and neuromuscular blockade Overall, $756(88.4 \%)$ of all patients received analgosedation. The use of propofol ( $87.6 \%$ vs. $89.7 \%, 95 \%$ CI $0.91-1.05)$ and pentobarbital (2.3\% vs. $1 \%, 95 \%$ CI $0.63-9.21)$ was similar between the VAE and non-VAE groups. The use of dexmedetomidine ( $36.4 \%$ vs. $17.4 \%$, relative risk $2.09,95 \%$ CI $1.60-2.78)$, midazolam ( $13.9 \%$ vs. $4.1 \%$, relative risk 3.37 , 95\% CI 1.94-5.9), fentanyl (31.9\% vs. $10.9 \%$ relative risk 2.92(95\% CI 2.11-4.05), and neuromuscular blockade (15.5\% vs. $1.8 \%, 95 \%$ CI relative risk 8.66 [4.41-16.9] was higher in the patients who experienced a VAE (Table 1). 
Table 1 Characteristics of neurocritically ill patients $(N=855)$ admitted to the Neurocritical Care Service at Harborview Medical Center between January 1, 2014, and December 31, 2018, by VAE

\begin{tabular}{|c|c|c|c|}
\hline & $\begin{array}{l}\text { Mechanical ventilation } \\
\text { for } 3 \text { or more days with VAE } \\
(n=130)\end{array}$ & $\begin{array}{l}\text { Mechanical ventilation for } 3 \\
\text { or more days without VAE } \\
\text { ( } n=725)\end{array}$ & $\begin{array}{l}p \text { value }(95 \% \mathrm{Cl}) \text { or RR } \\
(95 \% \mathrm{Cl})\end{array}$ \\
\hline Age years mean (SD) & $52(18.4)$ & $56.6(17.03)$ & NS \\
\hline Sex (male) & $90(70 \%)$ & $439(61 \%)$ & RR 1.15(1.01-1.31) \\
\hline Body mass index mean (SD) & 29.8(8.6) & $27.9(7)$ & NS \\
\hline Diagnosis & & & $p<0.001$ \\
\hline Isolated traumatic brain injury & $26(16.8 \%)$ & 193(83.2\%) & \\
\hline Spontaneous subarachnoid hemorrhage & $25(14.2 \%)$ & $151(85.8 \%)$ & \\
\hline Non-traumatic intraparenchymal hemorrhage & $15(10.5 \%)$ & 128(89.5\%) & \\
\hline Traumatic spinal cord injury & $25(26.3 \%)$ & $70(73.7 \%)$ & \\
\hline Postoperative care in the Intensive care unit & $9(7.0 \%)$ & $81(11.2 \%)$ & \\
\hline Acute ischemic stroke & $5(8.3 \%)$ & $55(91.7 \%)$ & \\
\hline Status epilepticus & $7(25.9 \%)$ & 20(74.1\%) & \\
\hline Meningitis/encephalitis & $2(16.7 \%)$ & 10(83.3\%) & \\
\hline Others & $15(21.4 \%)$ & $11(78.6 \%)$ & \\
\hline Acute symptomatic hydrocephalus from shunt failure & $1(14.3 \%)$ & $6(85.7 \%)$ & \\
\hline Admission GCS median [range] & $9[3-15]$ & $7[3-15]$ & NS \\
\hline Intracranial pressure monitor $n(\%)$ & $40(31 \%)$ & $292(40.2 \%)$ & NS \\
\hline Maximum intracranial pressure mmHg mean (SD) & $39.6(23.7)$ & 29(18.4) & $p<0.001(5.3-15.8)$ \\
\hline Positive fluid balance during mechanical ventilation & 93(80.9\%) & $498(75 \%)$ & NS \\
\hline \multicolumn{4}{|l|}{ Analgosedation and neuromuscular blockade } \\
\hline Propofol & $87.6 \%$ & $89.7 \%$ & NS \\
\hline Dexmedetomidine & $36.4 \%$ & $17.4 \%$ & RR 2.09(1.6-2.8) \\
\hline Fentanyl & $31.9 \%$ & $10.9 \%$ & RR 2.92(2.11-4.05) \\
\hline Midazolam & $13.9 \%$ & $4.1 \%$ & RR 3.37(1.94-5.9) \\
\hline Pentobarbital & $2.3 \%$ & $1 \%$ & NS \\
\hline Neuromuscular blockade & $15.5 \%$ & $1.8 \%$ & RR 8.66(4.41-16.9) \\
\hline Average delivered FiO2\% mean (SD) & $53(13.3)$ & $47(13.1)$ & $p<0.001(3.6-8.7)$ \\
\hline Average PO2 mmHg mean (SD) & 113.8(34.3) & 138(48.9) & $p<0.001(14.9-33.5)$ \\
\hline Average PCO2 mmHg mean (SD) & $40.5(6.3)$ & $36.8(5.6)$ & $p<0.001(2.63-4.86)$ \\
\hline Average $\mathrm{PaO} 2 / \mathrm{FiO} 2$ ratio mean (SD) & $256(184)$ & $329(238)$ & $p=0.0016(27.7-118.4)$ \\
\hline
\end{tabular}

FIO2 Fraction of inspired oxygen, GCS Glasgow Coma Score, NS not statistically significant, $P O 2$ partial pressure of oxygen, $P C O 2$ partial pressure of carbon dioxide, $\mathrm{PaO2} / \mathrm{FiO2}$ partial pressure of oxygen: fraction of inspired oxygen, $R R$ relative risk calculated for the association with occurrence of VAE, SD standard deviation, VAE ventilator-associated event

Fluid balance We did not observe any difference in the prevalence of positive fluid balance among patients with and without VAE ( $80.9 \%$ vs. $75 \%, p=$ not statistically significant).

\section{Microorganisms and Their Effect on Time to VAE, Duration of Mechanical Ventilation, and Intensive Care Unit Length of Stay}

The commonly occurring organisms were methicillinsusceptible staphylococcus aureus (28\%), Haemophilus species (13.2\%), methicillin-resistant staphylococcus aureus (11.8\%), Streptococcus species (9.2\%), Enterobacter species (7.9\%), Escherichia. coli (6.6\%), Klebsiella (6.6\%), Acinetobacter species (7.9\%), Moraxella catarrhalis $(2.6 \%)$, and Proteus species (2.6\%). There was no statistical difference in the prevalence of one particular organism over another in patients with and without VAE. There was no difference in the duration of mechanical ventilation and no difference in the intensive care unit length of stay between various microorganisms among patients in the VAE and non-VAE groups.

\section{Differences in Delivered FiO2, PO2, PCO2, and P/F Ratios in Patients with and without VAE}

The delivered $\mathrm{FiO} 2$ was $6 \%$ higher in the VAE group (53[SD 13.3] \% vs. 47[SD 13] \%, $p<0.00195 \%$ CI 3.68.7. The average $\mathrm{PO} 2$ values were $24.2 \mathrm{mmHg}$ lower in patients with VAE 113.8 [SD 34.3] $\mathrm{mmHg}$ vs. 138 [SD 34.3]. $p<0.001,95 \%$ CI 14.9-33.5). 
The average PCO2 values were $3.7 \mathrm{mmHg}$ higher in patients with VAE (40.5[SD 6.3] mmHg vs. 36.8[SD 5.6], $p<0.00195 \%$ CI 2.63-4.86).
The average $P / F$ ratio was $103 \mathrm{mmHg}$ lower in patients with VAE (256[SD 184] vs. 329[SD 238], $p<0.001,95 \%$ CI 27.7-118.4).

Table 2 Rate of VAE in $\mathbf{8 5 5}$ neurocritically ill patients admitted for three or more days to Harborview Medical Center between January 1, 2014, and December 31, 2018

\begin{tabular}{|c|c|c|}
\hline Diagnosis and number of VAE events & Total ventilator days ${ }^{a}$ & $\begin{array}{l}\text { Rate of VAEs } \\
\text { per } 1000 \text { ventilator } \\
\text { days }\end{array}$ \\
\hline $\begin{array}{l}\text { Total VAE events }(n=147) \\
\text { VAE rate by diagnoses (total VAE events }=147)\end{array}$ & 11,319 & 13.0 \\
\hline Status epilepticus $(n=10)$ & 370.6 & 26.98 \\
\hline Traumatic spinal cord injury $(n=30)$ & 1514.8 & 19.80 \\
\hline Hydrocephalus $(n=2)$ & 105.4 & 18.98 \\
\hline Others $(n=3)$ & 189.5 & 15.83 \\
\hline Meningitis/encephalitis $(n=2)$ & 133.7 & 14.96 \\
\hline Isolated traumatic brain injury $(n=42)$ & 2905.4 & 14.46 \\
\hline Spontaneous subarachnoid hemorrhage $(n=25)$ & 2022.8 & 12.36 \\
\hline Non-traumatic intraparenchymal hemorrhage $(n=19)$ & 1801.3 & 10.55 \\
\hline Acute ischemic stroke $(n=6)$ & 807.9 & 7.43 \\
\hline Postoperative care in the intensive care unit $(n=8)$ & 1432.5 & 5.58 \\
\hline
\end{tabular}

VAE ventilator-associated event

${ }^{a}$ Total ventilator days only include patients with three or more days on the ventilator

Table 3 Type of VAE $(n=147)$ and trigger for VAEs in 130 patients who received mechanical ventilation for three or more days

\begin{tabular}{llll} 
& Total number of VAEs & \multicolumn{1}{l}{ Trigger for VAEs } \\
\cline { 3 - 4 } & $n=147$ & $\begin{array}{l}\text { FiO2 } \\
n=24\end{array}$ & $\begin{array}{l}\text { PEEP } \\
n=123\end{array}$ \\
Ventilator-associated condition (VAC) & & $13(15.3 \%)$ & $72(84.7 \%)$ \\
Infection-related VAC (IVAC) & $85(58 \%)$ & $6(15.2 \%)$ & $27(81.8 \%)$ \\
Possible ventilator-associated pneumonia (PVAP) & $33(22 \%)$ & $4(13.8 \%)$ & $25(86.2 \%)$
\end{tabular}

FiO2 fraction of inspired oxygen, IVAC infection-related ventilator associated condition, PEEP positive end-expiratory pressure, PVAP possible ventilator associated pneumonia, VAC ventilator-associated condition, VAE ventilator-associated event

Table 4 Effect of VAE on clinical outcomes in 855 neurocritically ill patients admitted to Harborview Medical Center between January 1, 2014, and December 31, 2018

\begin{tabular}{|c|c|c|c|}
\hline & $\begin{array}{l}\text { Mechanical ventilation For } 3 \text { or } \\
\text { more days without VAE } \\
(n=725)\end{array}$ & $\begin{array}{l}\text { Mechanical ventilation } \\
\text { For } 3 \text { or more days with VAE } \\
(n=130)\end{array}$ & $p$ value $(95 \% \mathrm{Cl})$ \\
\hline $\begin{array}{l}\text { Duration of mechanical ventilation days, median [interquar- } \\
\text { tile range] }\end{array}$ & $7.9[8.9]$ & $17.4[20.5]$ & $<0.001(7.86-13.9)$ \\
\hline $\begin{array}{l}\text { Intensive care unit length of stay days, median [interquartile } \\
\text { range] }\end{array}$ & $12.5[0.4]$ & $20.2[1.1]$ & $<0.001(5.3-10.02)$ \\
\hline Hospital length of stay days, median [interquartile range] & $26.5[1.2]$ & $29.3[2.8]$ & NS \\
\hline In-hospital mortality & $31.3 \%$ & $34.1 \%$ & NS \\
\hline Transition to comfort measures only ${ }^{\mathrm{a}}$ & $70.9 \%$ & $52.6 \%$ & NS \\
\hline Discharge to home & $16.3 \%$ & $12.7 \%$ & NS \\
\hline
\end{tabular}

VAE ventilator-associated event

a The numerator is all patients transitioned to comfort measures only, denominator is all patients who experienced in-hospital mortality 


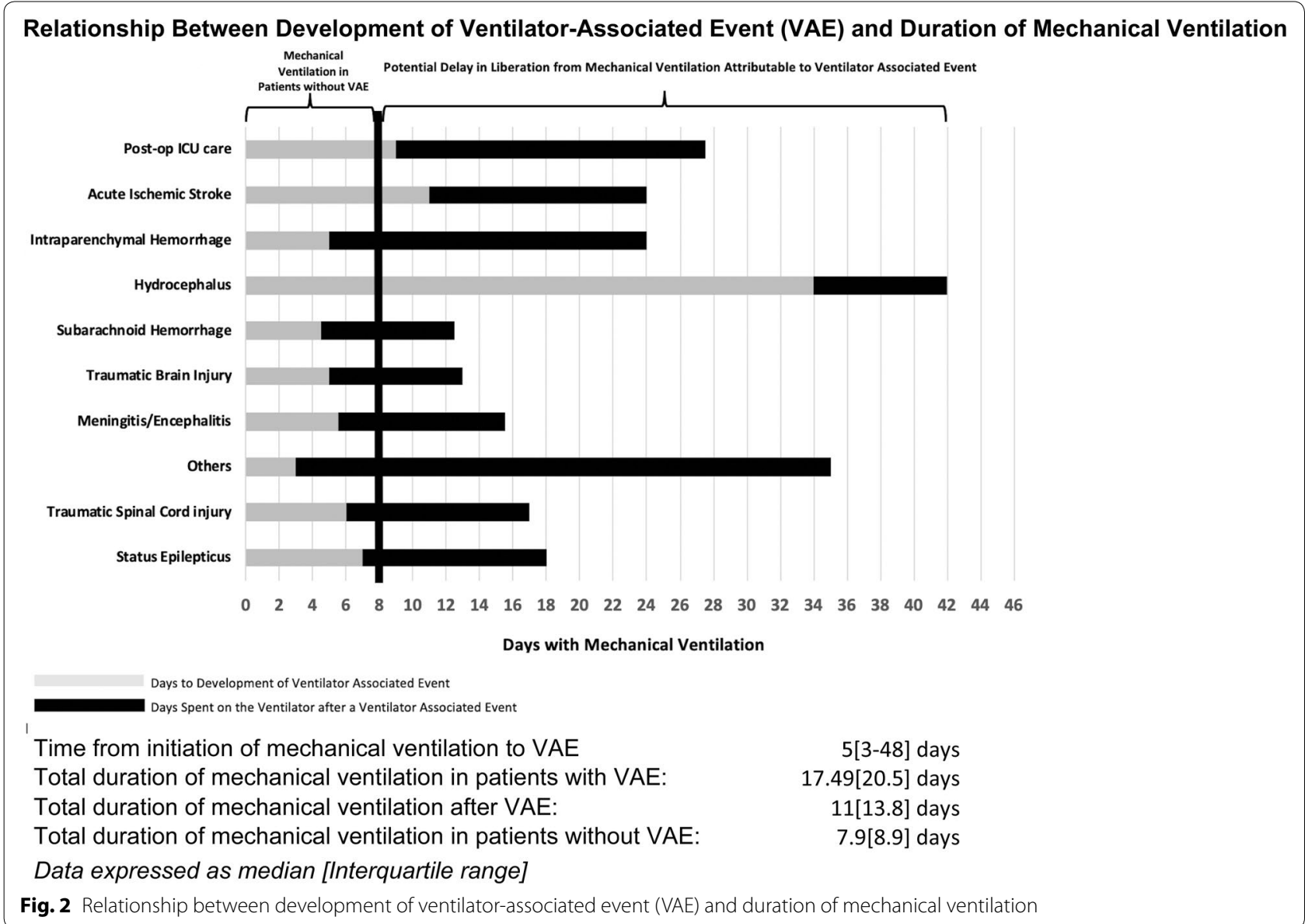

Impact of VAE on the Duration of Mechanical Ventilation Time from initiation of mechanical ventilation to the development of VAE was 5(3-48) days. Patients with VAE spent a long time on mechanical ventilation (17.4[20.5] vs. 7.9[8.9] days, 95\% CI 7.86-13.9) (Table 4). Time from VAE to liberation from mechanical ventilation was 11(13.8) days. Thus, this time was greater than the total duration of mechanical ventilation in patients without VAE, as highlighted in Fig. 2 and Supplementary Table 1.

\section{Association Between VAE and Outcomes}

Patients with VAE experienced longer intensive care unit stay $(20.2[1.1]$ vs. $12.5[0.4]$ days, $p<0.00195 \% \mathrm{CI}$ 5.3-10.02).

Mortality (34.1\% vs. $31.3 \%$, 95\% CI $0.84-1.41)$, transition to comfort measures only $(52.6 \%$ VS. $70.2 \%, 95 \%$ CI 0.54-1.01), median hospital length of stay (29.3[2.8] vs. 26.5[1.2] days, 95\% CI -9.2-1.76), and discharge to home $(12.7 \%$ vs. $16.3 \%, 0.48-1.29)$ were similar in patients with and without VAE (Table 4).

\section{Discussion}

The main findings of this study are that (1) VAEs are prevalent among neurocritically ill patients, occur within the first week of mechanical ventilation, across age, sex, BMI, and GCS, and the rates differ among various diagnoses, (2) the most common trigger for VAE is an increase in PEEP, and (3) VAE is associated with increased time spent on mechanical ventilation but is not associated with increased in-hospital mortality or discharge to home.

\section{Prevalence of VAE in Neurocritical Care}

This study finds that VAEs are prevalent among the neurocritically ill and that the rates differ among various conditions. The rates of VAE reported in the literature range from 5.8 to 49.9 per 1000 ventilator days [8-10]. The rates previously expressed among the neurologically impaired patients are between 4.9 and 8.9 per 1000 ventilator days, which is lower than the 13 per 1000 ventilator days seen in our population $[5,9]$. It is unclear from prior studies whether the isolated traumatic brain and spinal 
cord injured patients were among those studied; therefore, these results may not be comparable.

The time from initiation of mechanical ventilation to VAE reported in this study is similar to a previously published study [11], and it is important to note that VAE frequently occurs in the first week of initiation of mechanical ventilation, corresponding to the time the brain is most susceptible to secondary brain injury.

This study provides new information related to rates of VAE among various neurocritical conditions. The occurrence of VAE among patients with subarachnoid hemorrhage, intracerebral hemorrhage, acute ischemic stroke, status epilepticus, and isolated brain injury may highlight reactionary changes in PEEP and $\mathrm{FiO} 2$ changes as acute lung injury or acute lung injury or acute respiratory distress syndrome develops commonly in these conditions [12-18]. In summary, VAEs are prevalent, occur mostly during the first week of illness, and are present across various neurological conditions.

\section{Proposed Association Between Isolated Traumatic Brain,} Isolated Spinal Cord Injury, and Status Epilepticus and VAE

Patients with isolated traumatic brain injury, especially those with severe injury, may be at risk of VAP due to the concomitant presence of intracranial hypertension. This finding supports evidence from a previous study by Lou et al. [19] that examined the relationship between intracranial hypertension, acute lung injury, and systemic inflammatory response syndrome. In preclinical models, it has been demonstrated that even in the absence of hypoperfusion or hypoxemia, acute intracranial hypertension was associated with higher scores for inflammation, edema, and necrosis in heart, lung, kidney, and liver tissues [20]. There is increase in neuron-specific enolase, tumor necrosis factor-alpha plasma concentrations, extravascular lung water, and lung density [21]. In addition, severe traumatic brain injury also predisposes patients to myocardial dysfunction, which can present as reduced ejection fraction and may likely precipitate volume overload, and potential hypoxemic respiratory failure.

This implies that critical care management of patients with intracranial hypertension is often complicated by pulmonary dysfunction, which in turn may negatively affect cerebral oxygenation, and VAE may reflect a clinical response to reduce the burden of secondary brain injury.

Patients with isolated spinal cord injury may be exquisitely susceptible to VAE events. Proposed mechanisms include proportional reduction in functional residual capacity and forced vital capacity with increasing level of spinal cord injury involving the cervical segment. This predisposes patients to reduced cough effort, retained secretions, atelectasis, alveolar hypoventilation, pulmonary shunting, and hypoxia. Either of these conditions may require changes in PEEP and or FiO2, which will affect VAE rates.

Patients with status epilepticus may be at multifactorial higher risk of VAE. Proposed mechanisms include aspiration of gastric contents during ictus complicating subsequent ventilator management, the use of intravenous anesthetic agents such as propofol, barbiturate and midazolam infusion, level of sedation desired to interrupt the process of status epilepticus, and its downstream effects on the overall duration of mechanical ventilation, propensity for hospital-acquired infection. In addition, fluid resuscitation in patients with status epilepticus who experience rhabdomyolysis may also affect the VAE rate due to volume overload.

\section{Impact of VAE on Liberation from Mechanical Ventilation} Our study finds that the liberation from mechanical ventilation is delayed in patients who experience VAE. This finding is similar to the previous study on non-neurocritically ill patients [22]. Altered mental status may be one [23] of the many factors precluding extubation in neurologically impaired patients; however, the fact that the admission GCS was higher in patients with VAE may imply that successful weaning from mechanical ventilation is poorly correlated with the level of consciousness.

\section{Impact of VAE on Clinical Outcomes}

Outcomes, such as mortality, median hospital length of stay, and discharge to home were not affected by VAE. This is contradictory to prior studies on non-neurologically critically ill patients, which demonstrated that VAE patients are twice as likely to die than non-VAE patients [24]. These studies did not indicate the specific mechanism of injury, and it is possible that in certain injury mechanisms (adult repiratory disease syndrome, polytrauma, sepsis), VAE could signify as a marker of injury severity. Although we did not find statistical differences in overall mortality, the impact of VAE on the transition to comfort measures only deserves further examination in a larger cohort of patients.

\section{Implications on VAE in Neurocritical Care and Unanswered Questions}

In our hospital, VAE are abstracted from the electronic medical records as a quality improvement measure, and neurocritical care clinicians do not have access to this information during routine care of the patient. The VAE terminology was initially developed for surveillance purposes and does not have an ICD code. VAE may, in fact, reflect reactionary measures taken by practitioners as they manage patients with acute lung injury or adult 
respiratory distress syndrome as part of the "PEEP ladder." Non-infectious VAE, which comprise the majority of VAEs, both in our study and in previous studies, may be attributed to pulmonary edema, or atelectasis, and not necessarily to pneumonia, and VAP may occur without being preceded by a VAE.

Factors such as input/output balance $\geq 50 \mathrm{ml}$, gastric residuals $\geq 200 \mathrm{ml}$, and prolonged analgosedation have been identified as being associated with VAE [25]. Contrary to belief, compliance with ventilator bundle and chlorhexidine oral care has been shown to be associated with increased incidence of VAE [26]. Spontaneous breathing and awakening trials have been shown to reduce the overall incidence of $\operatorname{VAE}[27,28]$.

In our hospital, aggressive ventilator weaning in the form of a reduction in PEEP and $\mathrm{FiO} 2$ is standard of care. It may be possible that this strategy may potentially increase VAE rates, and it is possible for VAE rates to differ by institutional variability depending on how mechanical ventilation parameters are adjusted [29].

In patients with intracranial hypertension, secondary brain injury, and systemic inflammatory response that ensues can manifest in the form of VAE. Frequently, patients with intracranial hypertension patients are receiving deep sedation often for prolonged periods, which in itself may be a factor associated with VAE.

In addition, leukocytosis and elevated temperature are common findings in the neurocritically ill patients, and the commencement of antibiotics is by no means diagnostic of infection. These are potential drawbacks of the CDC-NHSN criteria.

Thus, can VAE truly be preventable? These questions deserve further examination in larger cohorts of neurocritically ill patients within a specific diagnosis group.

\section{Limitations}

This single-center retrospective cohort study has some strengths and limitations. Since the overall rates of VAE were small, it precluded any analysis of patients with specific clinical conditions. However, the retrospective design reduces the possibility of manipulating ventilator settings to evade VAE diagnosis, which has been a concern regarding the validity of VAE rates in other studies [30]. If patients were maintained on higher levels of $\mathrm{FiO} 2$ than physiologically necessary, this could have potentially masked VAEs that would have met criteria by an increase in $\mathrm{FiO} 2$, making our results more significant despite this handicap. Due to the small sample size of VAE, we were unable to examine the association between patient characteristics and the development of VAE. Finally, the incidence of VAE based on diagnosis was derived from small numbers of actual VAE events for many diagnoses. These incidences should be used with caution until they can be further validated in larger studies.

\section{Conclusions}

VAE are prevalent among neurocritically ill patients, with the rates differing among various neurocritical care conditions. Presence of a VAE may be associated with increased time spent on mechanical ventilation, and in the intensive care unit, but may not be associated with mortality or discharge to home.

\section{Electronic supplementary material}

The online version of this article (https://doi.org/10.1007/s12028-019-00910-5) contains supplementary material, which is available to authorized users.

\section{Author details}

${ }^{1}$ School of Medicine, University of Washington, Seattle, WA, USA. ${ }^{2}$ Department of Anesthesiology and Pain Medicine, University of Washington, Seattle, WA, USA. ${ }^{3}$ Neurocritical Care Service, Neuroscience ICU, Seattle, USA. ${ }^{4}$ Department of Neurological Surgery, University of Washington, Seattle, WA, USA. ${ }^{5}$ Associate Faculty, Harborview Injury Prevention and Research Center, Harborview Medical Center, Seattle, WA 98104, USA.

\section{Author Contributions}

VKSW and CF contributed to study design, data collection, data interpretation and manuscript editing. AMW contributed to data collection, data interpretation and manuscript editing. AVL contributed to study design, data collection, data interpretation, statistical analysis and manuscript editing.

\section{Source of support}

AVL received research support from Aqueduct Critical Care and salary support from LifeCenter Northwest. All other authors have no financial disclosures to report.

\section{Conflicts of interest}

$A V L$ reports receiving research support from Aqueduct Critical Care and salary support from LifeCenter Northwest. The other authors do not have any conflicts of interest to report.

\section{Ethical Approval}

This study received approval from the Institutional Review Board of the University of Washington.

\section{Publisher's Note}

Springer Nature remains neutral with regard to jurisdictional claims in published maps and institutional affiliations.

Published online: 23 January 2020

\section{References}

1. Magill SS, Klompas M, Balk R, et al. Developing a new, national approach to surveillance for ventilator-associated events: executive summary. Clin Infect Dis. 2013;57(12):1742-6.

2. Meagher $A D$, Lind $M$, Senekjian $L$, et al. Ventilator-associated events, not ventilator-associated pneumonia, is associated with higher mortality in trauma patients. J Trauma Acute Care Surg. 2019;87(2):307-14.

3. Esteban A, Anzueto A, Frutos F, et al. Characteristics and outcomes in adult patients receiving mechanical ventilation: a 28-day international study. JAMA. 2002;287(3):345-55.

4. Wunsch H, Linde-Zwirble WT, Angus DC, Hartman ME, Milbrandt EB, Kahn JM. The epidemiology of mechanical ventilation use in the United States. Crit Care Med. 2010;38(10):1947-53. 
5. Centers for Disease Control. Pneumonia (Ventilator-associated [VAP] and non-ventilator-associated Pneumonia [PNEU]) Event. https://www. cdc.gov/nhsn/pdfs/pscmanual/6pscvapcurrent.pdf. Published 2019. Accessed 26 Aug 2019.

6. Centers for Disease Control. Ventilator-Associated Event (VAE). https:// www.cdc.gov/nhsn/pdfs/pscmanual/10-vae_final.pdf. Published 2019. Accessed 26 Aug 2019.

7. Stata/IC [computer program]. Version 15.1. College Station, TX: StataCorp LP; 2017.

8. Klompas M, Kleinman K, Murphy MV. Descriptive epidemiology and attributable morbidity of ventilator-associated events. Infect Control Hosp Epidemiol. 2014;35(5):502-10.

9. He S, Wu F, Wu X, et al. Ventilator-associated events after cardiac surgery evidence from 1,709 patients. J Thorac Dis. 2018;10(2):776-83.

10. Magill SS, Li Q, Gross C, Dudeck M, Allen-Bridson K, Edwards JR. Incidence and characteristics of ventilator-associated events reported to the National Healthcare Safety Network in 2014. Crit Care Med. 2016:44(12):2154-62.

11. Rello J, Ramirez-Estrada S, Romero A, et al. Factors associated with ventilator-associated events: an international multicenter prospective cohort study. Eur J Clin Microbiol Infect Dis. 2019;38(9):1693-9.

12. Veeravagu $A$, Chen $Y R$, Ludwig C, et al. Acute lung injury in patients with subarachnoid hemorrhage: a nationwide inpatient sample study. World Neurosurg. 2014;82(1-2):e235-41.

13. Cui JB, Chen QQ, Liu TT, Li SJ. Risk factors for early-onset ventilator-associated pneumonia in aneurysmal subarachnoid hemorrhage patients. Braz J Med Biol Res. 2018;51(7):e6830.

14. Mahdavi Y, Surges R, Nikoubashman O, et al. Neurogenic pulmonary edema following seizures: a retrospective computed tomography study. Epilepsy Behav. 2019;94:112-7.

15. Saracen A, Kotwica Z, Wozniak-Kosek A, Kasprzak P. Neurogenic pulmonary edema in aneurysmal subarachnoid hemorrhage. Adv Exp Med Biol. 2016:952:35-9.

16. Elmer J, Hou P, Wilcox SR, et al. Acute respiratory distress syndrome after spontaneous intracerebral hemorrhage. Crit Care Med. 2013;41(8):1992-2001.

17. Veeravagu A, Jiang B, Rincon F, Maltenfort M, Jallo J, Ratliff JK. Acute respiratory distress syndrome and acute lung injury in patients with vertebral column fracture(s) and spinal cord injury: a nationwide inpatient sample study. Spinal Cord. 2013;51(6):461-5.
18. Kahn JM, Caldwell EC, Deem S, Newell DW, Heckbert SR, Rubenfeld GD. Acute lung injury in patients with subarachnoid hemorrhage: incidence, risk factors, and outcome. Crit Care Med. 2006;34(1):196-202.

19. Lou M, Chen X, Wang K, Xue Y, Cui D, Xue F. Increased intracranial pressure is associated with the development of acute lung injury following severe traumatic brain injury. Clin Neurol Neurosurg. 2013;115(7):904-8.

20. Heuer JF, Selke M, Crozier TA, et al. Effects of acute intracranial hypertension on extracerebral organs: a randomized experimental study in pigs. J Neurol Surg A Cent Eur Neurosurg. 2012;73(5):289-95.

21. Heuer JF, Pelosi P, Hermann P, et al. Acute effects of intracranial hypertension and ARDS on pulmonary and neuronal damage: a randomized experimental study in pigs. Intensive Care Med. 2011;37(7):1182-91.

22. Sim JK, Oh JY, Min KH, et al. Clinical significance of ventilator-associated event. J Crit Care. 2016;35:19-23.

23. Karanjia N, Nordquist D, Stevens R, Nyquist P. A clinical description of extubation failure in patients with primary brain injury. Neurocrit Care. 2011;15(1):4-12.

24. Melsen WG, Rovers MM, Groenwold RH, et al. Attributable mortality of ventilator-associated pneumonia: a meta-analysis of individual patient data from randomised prevention studies. Lancet Infect Dis. 2013;13(8):665-71.

25. Liu J, Zhang S, Chen J, et al. Risk factors for ventilator-associated events: a prospective cohort study. Am J Infect Control. 2019;47(7):744-9.

26. Harris BD, Thomas GA, Greene MH, Spires SS, Talbot TR. Ventilator bundle compliance and risk of ventilator-associated events. Infect Control Hosp Epidemiol. 2018;39(6):637-43.

27. Cocoros NM, Klompas M. Ventilator-associated events and their prevention. Infect Dis Clin North Am. 2016;30(4):887-908.

28. Klompas $M$, Anderson $D$, Trick $W$, et al. The preventability of ventilatorassociated events. The CDC prevention epicenters wake up and breathe collaborative. Am J Respir Crit Care Med. 2015;191(3):292-301.

29. Nair GB, Niederman MS. Using ventilator-associated pneumonia rates as a health care quality indicator: a contentious concept. Semin Respir Crit Care Med. 2017;38(3):237-44.

30. Lilly CM, Landry KE, Sood RN, et al. Prevalence and test characteristics of national health safety network ventilator-associated events. Crit Care Med. 2014;42(9):2019-28. 